\title{
Stability of Delay Impulsive Systems with Application to Networked Control Systems
}

\author{
Payam Naghshtabrizi, João P. Hespanha, and Andrew R. Teel
}

\begin{abstract}
We establish asymptotic and exponential stability theorems for delay impulsive systems by employing Lyapunov functionals with discontinuities. Our conditions have the property that when specialized to linear delay impulsive systems, the stability tests can be formulated as Linear Matrix Inequalities (LMIs). Then we consider Networked Control Systems (NCSs) consisting of an LTI process and a static feedback controller connected through a communication network. Due to the shared and unreliable channels, sampling intervals are uncertain and variable. Moreover, samples may be dropped and experience uncertain and variable delays before arriving at the destination. We show that the resulting NCSs can be modeled by linear delay impulsive systems and we provide conditions for stability of the closed-loop in terms of LMIs. By solving these LMIs, one can find a positive constant that determines an upper bound between a sampling time and the subsequent input update time, for which stability of the closed-loop system is guaranteed.
\end{abstract}

\section{INTRODUCTION}

Impulsive dynamical systems exhibit continuous evolutions described by Ordinary Differential Equations (ODEs) and instantaneous state jumps or impulses. Motivated by systems with delay, we are interested in studying delay impulsive systems. We establish stability, asymptotic stability, and exponential stability theorems for delay impulsive systems by employing functionals with discontinuities at a countable set of times.

By defining the time lag space and other related concepts, criteria for the uniform stability and uniform asymptotic stability for Hybrid Dynamical Systems (HDSs) with time delays are constructed in [1], [2] using Razumikhin's Theorem. The same authors apply these results to analyze the stability of impulsive delay differential equations and nonlinear sampled-data feedback control systems with time delay. Michel et al. [3] presents Lyapunov-Krasovskii type stability results and converse theorems for HDSs with time delay. Based on Lyapunov-Krasovskii functionals, [4] analyzes a class of HDSs consisting of delay differential equations with discontinuities. The authors consider a unified framework for a wide classes of HDSs and provide different types of stability and converse theorems by employing a positive definite discontinuous functional. If the functional is bounded between discontinuities and "appropriately" decreases at the point of discontinuities then one obtains an "appropriate"

P. Naghshtabrizi, J.P. Hespanha, and A. R. Teel are with the Department of Electrical \& Computer Engineering, University of California, Santa Barbara, CA 93106-9560, U.S.A.

Naghshtabrizi and Hespanha were supported by the Institute for Collaborative Biotechnologies through grant DAAD19-03-D-0004 from the U.S. Army Research Office and by the National Science Foundation under Grant No. CCR-0311084. Teel was supported by the Air Force Office of Scientific Research under Grant no. FA9550-06-1-0134, and National Science Foundation under Grant No. ECS-0622253

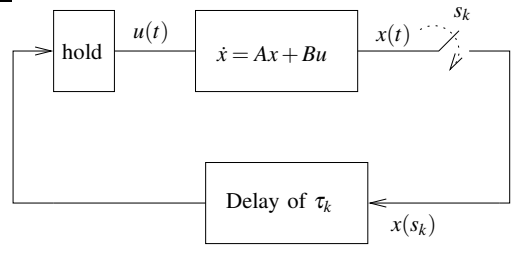

Fig. 1. NCSs with delay in the feedback loop where $u(t)=x\left(s_{k}\right), \forall t \in$ $\left[s_{k}+\tau_{k}, s_{k+1}+\tau_{k+1}\right)$

notion of stability (such as uniform stability, asymptotic stability or exponential stability).

A distinguishing feature of the stability conditions in this paper is that, when specialized to linear impulsive systems, the stability tests can be formulated as LMIs, which can be solved efficiently. Another advantage of our results over those in [4], [3] is that to verify the conditions in this paper, one does not need to compute the solution of the system between discontinuities: We only require a negative derivative of the Lyapunov functional along the solution and non-increase of the functional at the points of discontinuity.

As a special case of general delay impulsive systems, we study linear delay impulsive systems such as the one in Fig. 1, which can be expressed by

$$
\dot{x}(t)=A x(t)+B x\left(s_{k}\right), \quad t_{k} \leq t<t_{k+1}, k \in \mathbb{N},
$$

where $s_{k}$ denotes the $k$-th sampling time and $t_{k}$ the so called $k$-th input update time, which is the time instant at which the $k$-th sample arrives to the destination. In particular, denoting by $\tau_{k}$ the total delay that the $k$-th sample experiences in the loop, then $t_{k}:=s_{k}+\tau_{k}$. Fig. 1 and equation (1) can be viewed as modeling an NCS in which a linear process $\dot{x}(t)=$ $A x(t)+B_{u} u(t)$ is in feedback with a static state-feedback remote controller with gain $K$. This would correspond to $B:=B_{u} K$ in (1).

We introduce a new discontinuous Lyapunov functional to establish the stability of (1) based on the theorems developed here for general nonlinear time-varying delay impulsive systems. The Lyapunov functional is discontinuous at the input update times, but a decrease is guaranteed by construction. We provide an inequality that guarantees the decrease of the Lyapunov functional between the discontinuities, from which stability follows. This inequality is expressed as a set of LMIs that can be solved numerically using software packages such as MATLAB. By solving these LMIs, one can find a positive constant that determines an upper bound between the sampling time $s_{k}$ and the next input update time $t_{k+1}$, for which the stability of the closed-loop system is guaranteed for given lower and upper bounds on the total delay $\tau_{k}$. When there is no delay, this upper bound corresponds to 
the maximum sampling interval, which is often called $\tau_{M A T I}$ in the NCS literature. We use the $\tau_{M A T I}$ terminology also for the case when there are delays in the system, which allows us to state our result in the form: the system (1) is exponentially stable for any sampling-delay sequence satisfying $s_{k+1}+\tau_{k+1}-s_{k} \leq \tau_{M A T I}$ and $\tau_{\min } \leq \tau_{k} \leq \tau_{\max }$ for $\forall k \in \mathbb{N}$, where $\tau_{\min }, \tau_{\max }$, and $\tau_{\text {MATI }}$ appears in our LMIs.

To reduce network traffic in NCSs, significant work has been devoted to finding values for $\tau_{\text {MATI }}$ that are not overly conservative (see [5] and references therein). First we review the related work in which there is no delay in the control loop. In [6], $\tau_{\text {MATI }}$ is computed for linear and nonlinear systems with Round-Robin (static) or Try-Once-Discard (TOD) (dynamic) protocols. Nesic etal. [7], [8] study the inputoutput stability properties of nonlinear NCSs based on a small gain theorem to find $\tau_{M A T I}$ for NCSs. [9], [10], [11] consider linear NCSs and formulate the problem of finding $\tau_{M A T I}$ as LMIs. In the presence of variable delays in the control loop [12], [13], [14] show that for a given lower bound $\tau_{\min }$ on the delay in the control loop, stability can be guaranteed for a less conservative $\tau_{\text {MATI }}$ than in the absence of the lower bound.

Our stability conditions depends both on the lower bound $\tau_{\min }$ and on an upper bound $\tau_{\max }$ on the loop delay, which can be estimated (perhaps conservatively) for most networks [15]. Through an example we show that considering a finite $\tau_{\max }$ can significantly reduce conservativeness. When the delay in the feedback loop is small $\left(\tau_{\min }, \tau_{\max } \rightarrow 0\right)$, our LMIs reduce to the ones presented in [11] which are less conservative than those in [9], [10]. This observation shows that the results in [11] are robust with respect to small delays.

In section II we present asymptotic and exponential stability tests for time-varying nonlinear delay impulsive systems. In section III we model NCSs as delay impulsive systems and we apply the theorems from section II to compute $\tau_{\text {MATI }}$. Section IV is devoted to conclusions and future work.

Notation: Given an interval $I \subset \mathbb{R}, B\left(I, \mathbb{R}^{n}\right)$ denotes the space of real functions from $I$ to $\mathbb{R}^{n}$ with norm $\|\phi\|:=$ $\sup _{t \in I}|\phi(t)|$, for all $\phi \in B\left(I, \mathbb{R}^{n}\right)$ where $|$.$| denotes any one of$ the equivalent norms in $\mathbb{R}^{n}$. For a given signal $x(\cdot), x_{t}$ denotes the function $x_{t}:[-r, 0] \rightarrow \mathbb{R}^{n}$ defined by $x_{t}(\theta)=x(t+\theta)$, $\forall \theta \in[-r, 0]$ for some positive constant $r$. In addition, $\dot{x}(t)$ denotes the right-hand side derivative of $x$ with respect to $t$, i.e., $\dot{x}(t)=\lim _{t^{\prime} \uparrow t} \frac{x\left(t^{\prime}\right)-x(t)}{t^{\prime}-t}$. A function $\alpha \in[0, \infty) \rightarrow[0, \infty)$ is of class $\mathscr{K}$, and we write $\alpha \in \mathscr{K}$ when $\alpha$ is continuous, strictly increasing, and $\alpha(0)=0$. If $\alpha$ is also unbounded, then we say it is of class $\mathscr{K}_{\infty}$ and we write $\alpha \in \mathscr{K}_{\infty}$. A (continuous) function $\beta:[0, \infty) \times[0, \infty) \rightarrow[0, \infty)$ is of class $\mathscr{K} \mathscr{L}$, and we write $\beta \in \mathscr{K} \mathscr{L}$ when, $\beta(., t)$ is of class $\mathscr{K}$ for each fixed $t \geq 0$ and $\beta(s, t)$ decreases to 0 as $t \rightarrow \infty$ for each fixed $s$.

\section{STABILITy OF DELAY IMPULSIVE SYSTEMS}

Consider the following delay impulsive system

$$
\begin{aligned}
\dot{x}(t) & =f_{k}(x(t), t), & & t_{k} \leq t<t_{k+1}, \\
x\left(t_{k}\right) & =g_{k}\left(x\left(s_{k}\right), t_{k}\right), & & k \in \mathbb{N},
\end{aligned}
$$

where $f_{k}$ and $g_{k}$ are locally Lipschitz functions [16] such that $f_{k}(0, t)=0, g_{k}(0, t)=0, \forall t \in \mathbb{R}_{[0, \infty)}$. For a given samplingdelay sequence $\left\{s_{k}, \tau_{k}: k \in \mathbb{N}\right\}$, the sequence of input update times $\left\{t_{k}, k \in \mathbb{N}\right\}$ is defined as $t_{k}:=s_{k}+\tau_{k}, k \in \mathbb{N}$. We call the system (2) a delay impulsive system since the reset map (2b) depends on the past value of state.

The sampling times $\left\{s_{0}, s_{1}, s_{2}, \cdots\right\}$ and the input update times $\left\{t_{0}, t_{1}, t_{2}, \cdots\right\}$ form unbounded strictly increasing sequences. We allow the delays $\tau_{k}$ to grow larger than the sampling intervals $s_{k}-s_{k-1}$, provided that the sequence of input update times $\left\{t_{0}, t_{1}, t_{2}, \cdots\right\}$ remains strictly increasing. In essence, this means that if a sample gets to the destination out of order (i.e., an old sample gets to the destination after the most recent one), it should be dropped. Samples that do not reach the destination because of communication faults, or get dropped at the destination are simply not indexed, which allow us to model the effect of packet dropouts in the NCSs setting [10].

We can view (2) as an infinite dimensional system whose state contains the past history of $x(\cdot)$ so that $x\left(s_{k}\right)$ can be recovered from the state $x_{t_{k}}$ in order to apply the reset map in (2b). This allow us to apply Lyapunov-Krasovskii tools in the analysis of (2). In this framework, it is straightforward to analyze (2) even when the delays grows much larger than the sampling intervals, which is not easy in methods based on a discretization of (2) between update times [17], [18].

We assume that the impulse-delay sequences $\left\{s_{k}, \tau_{k}\right\}$ belong to a given set $\mathscr{S}$ and consider different stability definitions for (2) over $\mathscr{S}$ :

(a) The system (2) is said to be Globally Uniformly Stable (GUS) over $\mathscr{S}$, if there exists some $\alpha \in \mathscr{K}$ such that for every $\left\{s_{k}, \tau_{k}\right\} \in \mathscr{S}$ and every initial condition $x_{t_{0}}$ the solution to (2) is globally defined and satisfies $|x(t)| \leq \alpha\left(\left\|x_{t_{0}}\right\|\right), \forall t \geq$ $t_{0}$.

(b) The system (2) is said to be Globally Asymptotically Stable (GAS) over $\mathscr{S}$, if in addition to the conditions in (a), every solution converges to zero as $t \rightarrow \infty$.

(c) The system (2) is said to be Globally Uniformly Asymptotically Stable (GUAS) over $\mathscr{S}$, if there exists some $\beta \in \mathscr{K} \mathscr{L}$ such that for every $\left\{s_{k}, \tau_{k}\right\} \in \mathscr{S}$ and every initial condition $x_{t_{0}}$ the solution to (2) is globally defined and satisfies $|x(t)| \leq \beta\left(\left\|x_{t_{0}}\right\|, t-t_{0}\right), \forall t \geq t_{0}$.

(d) The system (2) is said to be Globally Uniformly Exponentially Stable (GUES) over $\mathscr{S}$, when the function $\beta$ in (c) is of the form $\beta(s, r)=c e^{-\lambda r} s$ for some $c, \lambda>0$.

Theorem 1: Suppose that there exist $\psi_{1}, \psi_{2} \in \mathscr{K}_{\infty}, \psi_{3} \in$ $\mathscr{K}$ and a functional $V: B\left([-r, 0], \mathbb{R}^{n}\right) \times \mathbb{R}_{[0, \infty)} \rightarrow \mathbb{R}_{[0, \infty)}$, absolutely continuous between input update times, such that

$$
\psi_{1}(|\phi(0)|) \leq V(\phi, t) \leq \psi_{2}(\|\phi\|), \forall \phi \in B\left(I, \mathbb{R}^{n}\right), t \geq 0,
$$

and, for every $\left\{s_{k}, \tau_{k}\right\} \in \mathscr{S}$, any solution $x$ to (2) is globally defined and satisfies

$$
\begin{array}{llrl}
\frac{\mathrm{d} V\left(x_{t}, t\right)}{\mathrm{d} t} & \leq-\psi_{3}(|x(t)|), & & t_{k} \leq t<t_{k+1}, k \in \mathbb{N}, \\
V\left(x_{t_{k}}, t_{k}\right) & \leq \lim _{t \uparrow t_{k}} V\left(x_{t}, t\right), & & \forall k \in \mathbb{N} .
\end{array}
$$


Then the system (2) is GUS over $\mathscr{S}$. In addition, the following statements hold:

(a) The system (2) is GUAS over $\mathscr{S}$ if there is a constant $h_{\min }>0$ for which $t_{k+1}-t_{k} \geq h_{\min }, \forall k \in \mathbb{N}$ for every $\left\{s_{k}, \tau_{k}\right\} \in \mathscr{S}$.

(b) The system (2) is UGES over $\mathscr{S}$, when the functions $\psi_{1}, \psi_{2}$ are of the following forms:

$$
\left.\psi_{1}(|\phi(0)|)\right):=c_{1}|\phi(0)|^{b}, \quad \psi_{2}(\|\phi\|):=c_{2}\|\phi\|^{b},
$$

and instead of (4), the following condition holds

$$
\frac{\mathrm{d} V\left(x_{t}, t\right)}{\mathrm{d} t} \leq-c_{3}\left\|x_{t}\right\|^{b}, \quad \forall t_{k} \leq t<t_{k+1}, k \in \mathbb{N}
$$

for some positive constants $c_{1}, c_{2}, c_{3}$, and $b$.

(c) The system (2) is UGES over $\mathscr{S}$, when the functions $\psi_{1}, \psi_{3}$ are of the following forms:

$$
\psi_{1}(|\phi(0)|):=d_{1}|\phi(0)|^{b}, \quad \psi_{3}(|x(t)|):=d_{3}|x(t)|^{b},
$$

and in (3) the upper bound $\psi_{2}(\|\phi\|)$ is replaced by

$$
d_{2}|\phi(0)|^{b}+\bar{d}_{2} \int_{t-r}^{t}|\phi(s)|^{b} d s,
$$

for some positive constants $d_{1}, d_{2}, \bar{d}_{2}, d_{3}$ and $b$.

Items (b) and (c) in Theorem 1 both provide alternative conditions to guarantee UGES over $\mathscr{S}$. The former poses milder conditions on the Lyapunov functional than the latter, but it poses a more strict condition on the time derivative of the functional. We shall see shortly that the latter statement will lead to sufficient conditions in terms of LMIs for linear impulsive systems.

Proof of Theorem 1. For every $\left\{s_{k}, \tau_{k}\right\} \in \mathscr{S}$, we have $\frac{\mathrm{d} V\left(x_{t}, t\right)}{\mathrm{d} t} \leq 0$ for $\forall t \in\left[t_{k}, t_{k+1}\right), k \in \mathbb{N}$, therefore

$$
\psi_{1}(|x(t)|) \leq V\left(x_{t}, t\right) \leq V\left(x_{t_{k}}, t_{k}\right), \quad t_{k} \leq t<t_{k+1} .
$$

Based on the conditions (4) and (5), we also have

$$
V\left(x_{t_{k+1}^{-}}, t_{k+1}^{-}\right) \leq V\left(x_{t_{k}}, t_{k}\right) \leq V\left(x_{t_{k}^{-}}, t_{k}^{-}\right), \quad k \in \mathbb{N},
$$

Combining (8) and (9), we conclude that

$$
\begin{aligned}
\psi_{1}(|x(t)|) \leq V\left(x_{t}, t\right) \leq V\left(x_{t_{k}^{-}}, t_{k}^{-}\right) \leq \cdots & \leq V\left(x_{t_{0}}, t_{0}\right) \\
& \leq \psi_{2}\left(\left\|x_{t_{0}}\right\|\right) .
\end{aligned}
$$

From (10), Lyapunov stability follows since $|x(t)| \leq$ $\alpha\left(\left\|x_{t_{0}}\right\|\right), \forall t \geq t_{0}$ for $\alpha():.=\psi_{1}^{-1}\left(\psi_{2}().\right)$.

(a) for every $\varepsilon>0$ let $\delta_{1}>0$ be such that $\psi_{2}\left(\delta_{1}\right) \leq \psi_{1}(\varepsilon)$. Then $\left\|x_{t_{0}}\right\| \leq \delta_{1}$ implies that $|x(t)|<\varepsilon, t \geq t_{0}$ because of (10). For this $\delta_{1}$ and any $\eta>0$, we show that there exists a $T=T\left(\delta_{1}, \eta\right)$ such that $|x(t)| \leq \eta$ for $\forall t \geq t_{0}+T$. Choose $\delta_{2}>0$ such that $\psi_{2}\left(\delta_{2}\right) \leq \psi_{1}(\eta)$ for $t \geq t_{0}+T$. Then it suffices to show that $\left\|x_{t_{0}+T}\right\|<\delta_{2}$ which implies $|x(t)|<$ $\eta, \forall t \geq 0$. By contradiction we assume that such a $T$ does not exist therefore there exists a sequence $c_{k}, k \in \mathbb{N}$ such that $\left\|x_{c_{k}}\right\|>\delta_{2}$. Each $c_{k}$ is in an interval $\left[t_{k_{i}}, t_{k_{i+1}}\right)$ where $t_{k_{i}}$ is a subsequence of $t_{k}$. Since $t_{k+1}-t_{k} \geq h_{\min }, \forall k \in \mathbb{N}$ then either $c_{k}-t_{k_{i}} \geq \frac{h_{\min }}{2}$ or $t_{k_{i+1}}-c_{k} \geq \frac{h_{\min }}{2}$. We define intervals

$$
I_{k}:=\left\{\begin{array}{ll}
{\left[c_{k}-\frac{\delta_{2}}{2 L_{1}}, c_{k}\right]} & \text { if } c_{k}-t_{k_{i}} \geq \frac{h_{\min }}{2} \\
{\left[c_{k}, c_{k}+\frac{\delta_{2}}{2 L_{1}}\right]} & \text { if } t_{k_{i+1}}-c_{k} \geq \frac{h_{\min }}{2}
\end{array},\right.
$$

where $L_{1}>\max \left(L, \frac{\delta_{2}}{h_{\min }}\right)$ and $\left|f_{k}(x, t)\right|<L$ for $\forall k \in \mathbb{N}$ (since $f_{k}$ is Lipshitz, there exists $L>0$ such that $\left.\left|f_{k}(x, t)\right|<L\right)$. By construction, $x(t)$ is continuous for any $t \in I_{k}$ and we can use the Mean Value Theorem. So for any $t \in I_{k}$ there exists a $\theta \in[0,1]$ such that

$$
\begin{aligned}
|x(t)| & =\left|x\left(c_{k}\right)+\dot{x}\left(c_{k}+\theta\left(t-c_{k}\right)\right)\left(t-c_{k}\right)\right| \geq\left|x\left(c_{k}\right)\right| \\
& -\left|\dot{x}\left(c_{k}+\theta\left(t-c_{k}\right)\right)\right|\left(\left|t-c_{k}\right|\right) \geq \delta_{2}-L \frac{\delta_{2}}{2 L_{1}} \geq \frac{\delta_{2}}{2} .
\end{aligned}
$$

Therefore $\frac{\mathrm{d} V\left(x_{t}, t\right)}{\mathrm{d} t} \leq-\psi_{3}\left(\frac{\delta_{2}}{2}\right)$ for any $t \in I_{k}$ and otherwise $\frac{\mathrm{d} V\left(x_{t}, t\right)}{\mathrm{d} t} \leq 0$. By integration we have

$$
V\left(x_{c_{k}}, c_{k}\right) \leq V\left(x_{t_{0}}, t_{0}\right)-\psi_{3}\left(\frac{\delta_{2}}{2}\right) \frac{k \delta_{2}}{2 L_{1}},
$$

but this would imply that $V\left(x_{c_{k}}, c_{k}\right)<0$ for a sufficiently large $k$. By contradiction, we conclude that the system is GUAS over $\mathscr{S}$.

(b) Inequalities (3) with the choice of (6) and (7) implies

$$
\frac{\mathrm{d} V\left(x_{t}, t\right)}{\mathrm{d} t} \leq-\frac{c_{3}}{c_{2}} V\left(x_{t}, t\right) .
$$

By the Comparison Lemma [16] we have $V\left(x_{t}, t\right) \leq$ $V\left(x_{t_{0}}, t_{0}\right) e^{-\frac{c_{3}}{c_{2}}\left(t-t_{0}\right)}$. Hence

$$
\begin{aligned}
|x(t)| & \leq\left(\frac{V\left(x_{t}, t\right)}{c_{1}}\right)^{1 / b} \leq\left(\frac{V\left(x_{t_{0}}, t_{0}\right) e^{-\frac{c_{3}}{c_{2}}\left(t-t_{0}\right)}}{c_{1}}\right)^{1 / b} \\
& \leq\left(\frac{c_{2}\left\|x_{t_{0}}\right\|^{b} e^{\frac{-c_{3}}{c_{2}}\left(t-t_{0}\right)}}{c_{1}}\right)^{1 / b}=\left(\frac{c_{2}}{c_{1}}\right)^{1 / b}\left\|x_{t_{0}}\right\| e^{-\frac{c_{3}}{c_{2} b}\left(t-t_{0}\right)} .
\end{aligned}
$$

Thus, the origin is GUES over $\mathscr{S}$.

(c) Defining $W\left(x_{t}, t\right):=e^{\varepsilon\left(t-t_{0}\right)} V\left(x_{t}, t\right)$, we conclude that

$$
\begin{aligned}
& \frac{\mathrm{d} W\left(x_{t}, t\right)}{\mathrm{d} t}=\varepsilon e^{\varepsilon\left(t-t_{0}\right)} V\left(x_{t}, t\right)+e^{\varepsilon\left(t-t_{0}\right)} \frac{\mathrm{d} V\left(x_{t}, t\right)}{\mathrm{d} t} \leq \\
& \varepsilon e^{\varepsilon\left(t-t_{0}\right)}\left(d_{2}|x(t)|^{b}+\bar{d}_{2} \int_{t-r}^{t}|x(v)|^{b} d v\right)-d_{3} e^{\varepsilon\left(t-t_{0}\right)}|x(t)|^{b} .
\end{aligned}
$$

By integration of (11), we have

$$
\begin{aligned}
& W\left(x_{t}, t\right)-W\left(x_{t_{0}}, t_{0}\right) \leq \varepsilon d_{2} \int_{t_{0}}^{t} e^{\varepsilon\left(s-t_{0}\right)}|x(s)|^{b} d s \\
& \quad+\varepsilon \bar{d}_{2} \int_{t_{0}}^{t} \int_{s-r}^{s} e^{\varepsilon\left(s-t_{0}\right)}|x(v)|^{b} d v d s-d_{3} \int_{t_{0}}^{t} e^{\varepsilon\left(s-t_{0}\right)}|x(s)|^{b} d s .
\end{aligned}
$$

One can show that

$$
\begin{aligned}
& \int_{t_{0}}^{t} \int_{s-r}^{s} e^{\varepsilon\left(s-t_{0}\right)}|x(v)|^{b} d v d s \leq \int_{t_{0}-r}^{t_{0}} \int_{t_{0}}^{v+r} e^{\varepsilon\left(s-t_{0}\right)}|x(v)|^{b} d s d v+ \\
& \int_{t_{0}}^{t} \int_{v}^{v+r} e^{\varepsilon\left(s-t_{0}\right)}|x(v)|^{b} d s d v \leq r e^{\varepsilon r} \int_{t_{0}-r}^{t_{0}}|x(v)|^{b} d v+ \\
& r e^{\varepsilon r} \int_{t_{0}}^{t} e^{\varepsilon\left(v-t_{0}\right)}|x(v)|^{b} d v .
\end{aligned}
$$

Combining (12), (13) and the fact that $W\left(x_{t_{0}}, t_{0}\right) \leq$ $d_{2}\left|x\left(t_{0}\right)\right|^{b}+\bar{d}_{2} \int_{t_{0}-r}^{t_{0}}|x(s)|^{b} d s$ we get

$$
\begin{aligned}
W\left(x_{t}, t\right) & \leq d_{2}\left|x\left(t_{0}\right)\right|^{b}+\bar{d}_{2}\left(1+\varepsilon r e^{\varepsilon r}\right) \int_{t_{0}-r}^{t_{0}}|x(v)|^{b} d v \\
& +\left(\varepsilon \bar{d}_{2} r e^{\varepsilon r}-d_{3}\right) \int_{t_{0}}^{t} e^{\varepsilon\left(v-t_{0}\right)}|x(v)|^{b} d v .
\end{aligned}
$$


For small enough $\varepsilon$,

$$
W\left(x_{t}, t\right) \leq d_{2}|x(0)|^{b}+\bar{d}_{2}\left(1+\varepsilon r e^{\varepsilon r}\right) \int_{t_{0}-r}^{t_{0}}|x(v)|^{b} d v .
$$

If (14) holds, there exists a $d_{4}>0$ such that $W\left(x_{t}, t\right) \leq$ $d_{4}\left\|x_{t_{0}}\right\|^{b}$ or $V\left(x_{t}, t\right) \leq d_{4} e^{-\varepsilon\left(t-t_{0}\right)}\left\|x_{t_{0}}\right\|^{b}$ and consequently $x(t) \leq\left(\frac{d_{4}}{d_{1}}\right)^{1 / b} e^{-\frac{\varepsilon}{b}\left(t-t_{0}\right)}\left\|x_{t_{0}}\right\|$ for every $\left\{s_{k}, \tau_{k}\right\} \in \mathscr{S}$.

\section{NCSS WITH VARIABLE SAMPLING AND DELAY}

Consider an NCS consisting of an LTI process with state space model of the form $\dot{x}(t)=A x(t)+B_{u} u(t), x \in \mathbb{R}^{n}, u \in$ $\mathbb{R}^{m}$ and a state-feedback controller with constant gain $K$ connected through sample and hold blocks. At time $s_{k}, k \in \mathbb{N}$ the process's state, $x\left(s_{k}\right)$, is sent to the controller and the control command $K x\left(s_{k}\right)$ is sent back to the process to be used as soon as it arrives, and until the next control command update. The total delay in the control loop that the $k$-th sample experiences is denoted by $\tau_{k}$. To be consistent with the results in [13], [14] and [12] we characterize the admissible set of sampling-delay sequences $\left\{s_{k}, \tau_{k}\right\}$ as

$$
\begin{aligned}
\mathscr{S}:=\left\{\left(s_{k}, \tau_{k}\right): s_{k+1}+\tau_{k+1}-s_{k}\right. & \leq \tau_{M A T I}, \\
\tau_{\min } & \left.\leq \tau_{k} \leq \tau_{\max }\right\} .
\end{aligned}
$$

The closed-loop NCS can be modeled by the following delay impulsive system

$$
\begin{aligned}
\dot{\xi}(t) & =F \xi(t), & & t_{k} \leq t<t_{k+1}, \\
\xi\left(t_{k+1}\right) & =\left[\begin{array}{c}
x\left(t_{k+1}^{-}\right) \\
x\left(s_{k+1}\right)
\end{array}\right], & & k \in \mathbb{N},
\end{aligned}
$$

where

$$
\begin{aligned}
F:=\left[\begin{array}{cc}
A & B \\
0 & 0
\end{array}\right], & \xi(t):=\left[\begin{array}{c}
x(t) \\
z_{1}(t)
\end{array}\right], \quad B:=B_{u} K, \\
t_{k}:=s_{k}+\tau_{k}, & z_{1}(t):=x\left(s_{k}\right), \quad t_{k} \leq t<t_{k+1} .
\end{aligned}
$$

Consider the Lyapunov functional

$$
\begin{aligned}
& V:=x^{\prime} P x+\int_{t-\rho_{1}}^{t}\left(\rho_{1 \max }-t+s\right) \dot{x}^{\prime}(s) R_{1} \dot{x}(s) d s+ \\
& \int_{t-\rho_{2}}^{t}\left(\rho_{2 \max }-t+s\right) \dot{x}^{\prime}(s) R_{2} \dot{x}(s) d s+ \\
& \int_{t-\tau_{\min }}^{t}\left(\tau_{\min }-t+s\right) \dot{x}^{\prime}(s) R_{3} \dot{x}(s) d s+ \\
& \int_{t-\rho_{1}}^{t-\tau_{\min }}\left(\rho_{1 \max }-t+s\right) \dot{x}^{\prime}(s) R_{4} \dot{x}(s) d s+ \\
& \left(\rho_{1 \max }-\tau_{\min }\right) \int_{t-\tau_{\min }}^{t} \dot{x}^{\prime}(s) R_{4} \dot{x}(s) d s+\int_{t-\tau_{\min }}^{t} x^{\prime}(s) Z x(s) d s+ \\
& \left(\rho_{1 \max }-\rho_{1}\right)\left(x-z_{2}\right)^{\prime} X\left(x-z_{2}\right),
\end{aligned}
$$

with $P, X, Z, R_{i}, i=1, . ., 4$ positive definite matrices and

$$
\begin{aligned}
z_{2}(t):=x\left(t_{k}\right), \rho_{1}(t) & :=t-s_{k}, \rho_{2}(t):=t-t_{k}, \quad t_{k} \leq t<t_{k+1}, \\
\rho_{1 \max } & :=\sup _{t \geq 0} \rho_{1}(t), \quad \rho_{2 \max }:=\sup _{t \geq 0} \rho_{2}(t) .
\end{aligned}
$$

If the LMIs in the next theorem are feasible for given $\beta_{\max }:=\tau_{M A T I}-\tau_{\min }, \tau_{\min }$, and $\tau_{\max }$, then there exists a $d_{3}>0$ such that $\frac{\mathrm{d} V\left(x_{t}, t\right)}{\mathrm{d} t} \leq-d_{3}|x(t)|^{2}$. It is straightforward to show that the Lyapunov functional (17) satisfies the remaining

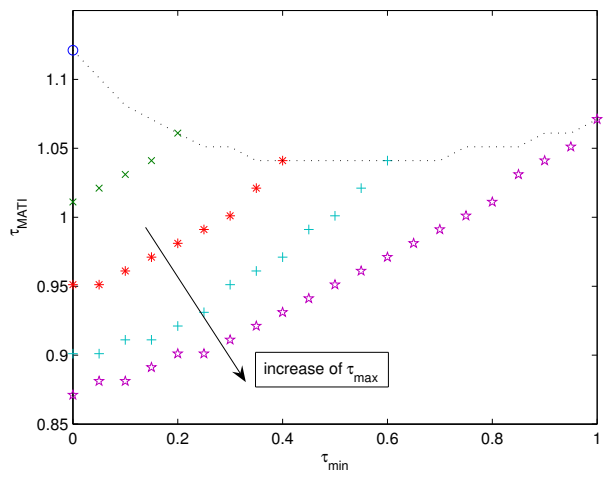

Fig. 2. $\tau_{\text {MATI }}$ versus $\tau_{\min }$ for $\tau_{\max }$ equal to $0, .2, .4, .6,1$ based on Theorem 2. The dashed line is the same as the one in Fig.3.

conditions in Theorem (1). Hence the NCS modeled by the delay impulsive system (16) is UGES over $\mathscr{S}$ given by (15).

Theorem 2: The system (16) is GUES over $\mathscr{S}$ defined by (15), provided that there exist positive definite matrices $P, X, Z, R_{i}, i=1, . ., 4$ and (not necessarily symmetric) matrices $N_{i}, i=1, . ., 4$ that satisfy the following LMIs:

$$
\begin{aligned}
& {\left[\begin{array}{ccc}
M_{1}+\beta_{\max }\left(M_{2}+M_{3}\right) & \tau_{\max } N_{1} & \tau_{\min } N_{3} \\
* & -\tau_{\max } R_{1} & 0 \\
* & * & -\tau_{\min } R_{3}
\end{array}\right]<0,} \\
& {\left[\begin{array}{ccccc}
M_{1}+\beta_{\max } M_{2} & \tau_{\max } N_{1} & \tau_{\min } N_{3} & \beta_{\max }\left(N_{1}+N_{2}\right) & \beta_{\max } N_{4} \\
* & -\tau_{\max } R_{1} & 0 & 0 & 0 \\
* & * & -\tau_{\min } R_{3} & 0 & 0 \\
* & * & * & -\beta_{\max }\left(R_{1}+R_{2}\right) & 0 \\
* & * & * & * & -\beta_{\max } R_{4}
\end{array}\right]<0,}
\end{aligned}
$$

where

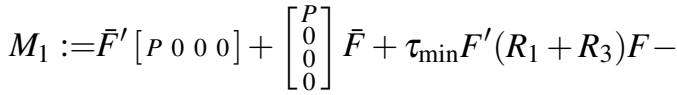

$$
\begin{aligned}
& {\left[\begin{array}{c}
I \\
0 \\
-I \\
0
\end{array}\right] X\left[\begin{array}{c}
I \\
0 \\
-I \\
0
\end{array}\right]^{\prime}+\left[\begin{array}{l}
I \\
0 \\
0 \\
0
\end{array}\right] Z\left[\begin{array}{l}
I \\
0 \\
0 \\
0
\end{array}\right]^{\prime}-\left[\begin{array}{l}
0 \\
0 \\
0 \\
I
\end{array}\right] Z\left[\begin{array}{l}
0 \\
0 \\
0 \\
I
\end{array}\right]^{\prime}}
\end{aligned}
$$

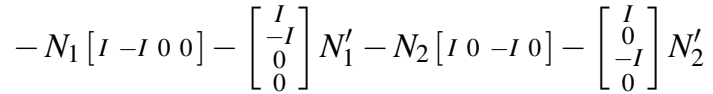

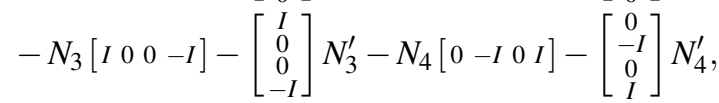

$$
\begin{aligned}
& M_{2}:=\bar{F}^{\prime}\left(R_{1}+R_{2}+R_{4}\right) \bar{F} \text {, } \\
& M_{3}:=\left[\begin{array}{c}
I \\
0 \\
-I \\
0
\end{array}\right] X \bar{F}+\bar{F}^{\prime} X\left[\begin{array}{llll}
I & 0 & -I & 0
\end{array}\right] .
\end{aligned}
$$

with $\bar{F}:=\left[\begin{array}{llll}A & B & 0 & 0\end{array}\right]$.

Remark 1: When the delays are small, i.e., $\tau_{\min }, \tau_{\max } \rightarrow 0$ the LMIs (18a) and (18b) are equivalent to

$$
\begin{aligned}
& M_{1}+\beta_{\max }\left(M_{2}+M 3\right)<0, \\
& M_{1}+\beta_{\max } M_{2}+\beta_{\max }\left(N_{1}+N_{2}\right)\left(R_{1}+R_{2}\right)^{-1}\left(N_{1}+N_{2}\right)^{\prime}<0
\end{aligned}
$$

(after using Schur Lemma). Making $R_{3}, R_{4}, Z, N_{3}, N_{4} \rightarrow 0$ and $N_{1}=\left[\begin{array}{llll}N_{11} & N_{12} & N_{13} & 0\end{array}\right]^{\prime}, \quad N_{2}=\left[\begin{array}{llll}N_{21} & N_{22} & N_{23} & 0\end{array}\right]^{\prime}$, makes the last row and column of the LMIs in (20) approach zero and we can omit them. After multiplying the LMIs in (1) by $\left[\begin{array}{lll}I & 0 & 0 \\ 0 & I & I\end{array}\right]$ and its transpose 


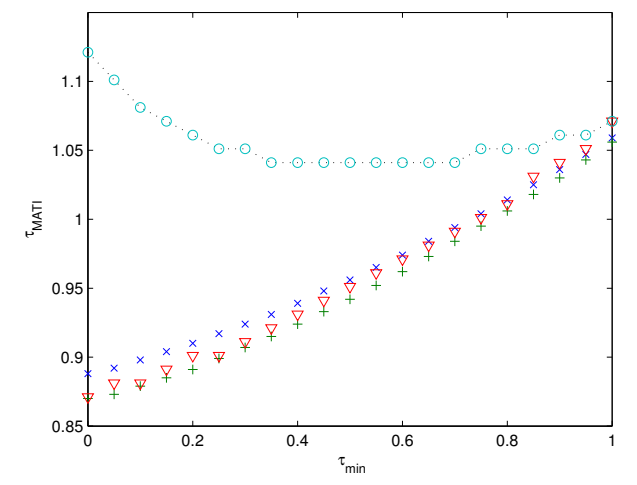

Fig. 3. $\tau_{\text {MATI }}$ versus $\tau_{\min }$ where $\tau_{\max }=\tau_{\min }$ from [13] ('+') and [14] (' $\times$ '), the worse case where $\tau_{\max }=\tau_{\text {MATI }}$ (' $\nabla$ ') and the best case where $\tau_{\max }=\tau_{\min }$ ('o') from Theorem 2 .

from the left and the right, respectively, choosing $N=$ $\left[\begin{array}{ll}N_{11}+N_{21} & N_{12}+N_{13}+N_{22}+N_{23}\end{array}\right]^{\prime}$ and $R_{1}+R_{2}=R$, we obtain the LMIs in [11, Theorem 1]. So the results in [11] are robust with respect to small delays, namely the results in [11] still guarantee stability for arbitrary small delays.

Example 1: Consider the state space process model [19]

$$
\left[\begin{array}{c}
\dot{x}_{1} \\
\dot{x}_{2}
\end{array}\right]=\left[\begin{array}{cc}
0 & 1 \\
0 & -0.1
\end{array}\right]\left[\begin{array}{l}
x_{1} \\
x_{2}
\end{array}\right]+\left[\begin{array}{c}
0 \\
0.1
\end{array}\right] u,
$$

with state feedback gain $K=-[3.7511 .5]$, for which we have

$$
A=\left[\begin{array}{cc}
0 & 1 \\
0 & -0.1
\end{array}\right], \quad B=-\left[\begin{array}{c}
0 \\
0.1
\end{array}\right] \times\left[\begin{array}{ll}
3.75 & 11.5
\end{array}\right] .
$$

By checking the condition eig $\left(\left[\begin{array}{ll}I & 0 \\ I & 0\end{array}\right] e^{F h}\right)<0$ on a tight grid of $h$, we can show that the closed-loop system remains stable for any constant sampling interval smaller than 1.7, and becomes unstable for larger constant sampling intervals. On the other hand, when the sampling interval approaches zero, the system is described by a DDE and we can find the maximum constant delay for which stability is guaranteed by looking at the roots of the characteristic function $\operatorname{det}(s I-$ $\left.A-B e^{-\tau_{0} s}\right)$. We use the Pade approximation $e^{-\tau_{0} s}=\frac{1-s \tau_{0} / 2}{1+s \tau_{0} / 2}$ to compute the determinant polynomial and conclude by the Routh-Hurwitz test that the system is stable for any constant delay smaller than 1.36. Comparing these numbers with the maximum variable sampling interval 1.1137 and the maximum variable delay 1.0744 both obtained using Theorem 2 (see below) reveals the conservativeness of our method:

No-delay and variable sampling: When there is no delay but the sampling intervals are variable, $\tau_{M A T I}$ determines an upper bound on the variable sampling intervals $s_{k+1}-s_{k}$ . The upper bound given by [9], [10], [13] (when $\tau_{\min }=0$ ) is 0.8696 which is improved to 0.8871 in [14]. Theorem 2 and [11] gives the upper bound equal to 1.1137 .

Variable-delay and sampling: Fig. 2 shows the value of $\tau_{\text {MATI }}$ obtained form Theorem 2, as a function of $\tau_{\min }$ for different values of $\tau_{\max }$. The dashed curves in Fig. 2 and Fig. 3 are the same which are comprised of the largest $\tau_{\text {MATI }}$ for different values of $\tau_{\max }$. Fig. 3 shows $\tau_{\text {MATI }}$ with respect to $\tau_{\min }$ where the results from [13], [14] are shown by,$+ \times$ respectively. The values of $\tau_{\text {MATI }}$ given by [12] lay between the " + " and " $x$ " in Fig. 3 and we do not show them. In Theorem 2, $\tau_{\text {MATI }}$ is a function of $\tau_{\min }$ and $\tau_{\max }$. To be able to compare our result to the others we consider two values for $\tau_{\max }$ and we obtain $\tau_{M A T I}$ as a function of $\tau_{\min }$ based on Theorem 2. Fist we consider $\tau_{\max }=\tau_{\min }$, which is the case that the delay is constant and equal to the value of $\tau_{\min }$. The largest $\tau_{\text {MATI }}$ for a given $\tau_{\text {min }}$ provided by Theorem 2 is shown using an "o" in Fig. 3. The second case is when $\tau_{\max }=\tau_{M A T I}$, which is the case where there can be very large delays in the loop in comparison to the sampling intervals. The largest $\tau_{\text {MATI }}$ for a given $\tau_{\min }$ for this case provided by Theorem 2 is shown using a " $\nabla$ " in Fig. 3. One can observe that when the delays in the control loop are small, our method shows a good improvement in comparison to the other results in the literature.

\section{CONCLUSIONS AND FUTURE WORK}

We established stability, asymptotic stability, and exponential stability theorems for delay impulsive systems. Our stability conditions have the property that when specialized to linear impulsive systems, the stability tests can be formulated as LMIs. Then we considered NCSs consisting of an LTI process and a static feedback controller connected through a communication network. Due to the shared, unreliable channel that connects process and controller, the sampling intervals and delays are uncertain and variable. We showed that the resulting NCSs can be modeled by linear delay impulsive systems. We provided conditions for the stability of the closed-loop expressed in terms of LMIs. By solving these LMIs, one can find a positive constant that determines an upper bound between the sampling time and the next input update time, for which stability of the closed-loop system is guaranteed.

Although in this paper we focused on the stability problem, it is possible to derive LMI conditions which lead to finding stabilizing static feedback gains (or $H_{\infty}$ design) [11]. We will extend our results to model more general NCSs such as two-channel NCSs with dynamic feedback controllers.

\section{APPENDIX}

Proof of Theorem 2. Along the trajectory of the system (2)

$$
\begin{aligned}
\dot{V}= & 2 x^{\prime}(t) P\left(A x(t)+B z_{1}\right)+\rho_{1 \max } \dot{x}^{\prime}(t) R_{1} \dot{x}(t) \\
& -\int_{t-\rho_{1}}^{t} \dot{x}^{\prime}(s) R_{1} \dot{x}(s) d s+\rho_{2 \max } \dot{x}^{\prime}(t) R_{2} \dot{x}(t) \\
& -\int_{t-\rho_{2}}^{t} \dot{x}^{\prime}(s) R_{2} \dot{x}(s) d s+\tau_{\min } \dot{x}^{\prime}(t) R_{3} \dot{x}(t) \\
& -\int_{t-\tau_{\min }}^{t} \dot{x}^{\prime}(s) R_{3} \dot{x}(s) d s \\
& +\left(\rho_{1 \max }-\tau_{\min }\right)\left(\dot{x}^{\prime}\left(t-\tau_{\min }\right) R_{4} \dot{x}\left(t-\tau_{\min }\right)\right) \\
& -\int_{t-\rho_{1}}^{t-\tau_{\min }} \dot{x}^{\prime}(s) R_{4} \dot{x}(s) d s+\left(\rho_{1 \max }-\tau_{\min }\right)\left(\dot{x}^{\prime}(t) R_{4} \dot{x}(t)\right. \\
& \left.-\dot{x}^{\prime}\left(t-\tau_{\min }\right) R_{4} \dot{x}\left(t-\tau_{\min }\right)\right)+x^{\prime}(t) Z x(t) \\
& -x^{\prime}\left(t-\tau_{\min }\right) Z x\left(t-\tau_{\min }\right)-\left(x(t)-z_{2}\right)^{\prime} X\left(x(t)-z_{2}\right) \\
& +2\left(\rho_{1 \max }-\rho_{1}\right)\left(x-z_{2}\right)^{\prime} X\left(A x+B z_{1}\right) .
\end{aligned}
$$


Defining $\bar{\xi}(t):=\left[\begin{array}{llll}x^{\prime}(t) & z_{1}^{\prime} & z_{2}^{\prime} & x^{\prime}\left(t-\tau_{\min }\right)\end{array}\right]^{\prime}$, for any matrices $N_{i}, i=1, \ldots, 4$ we have

$$
\begin{aligned}
& 2 \bar{\xi}^{\prime} N_{1}\left[\begin{array}{llll}
I & -I & 0 & 0
\end{array}\right] \bar{\xi}+2 \bar{\xi}^{\prime} N_{2}\left[\begin{array}{llll}
I & 0 & -I & 0
\end{array}\right] \bar{\xi} \\
& =2 \bar{\xi}^{\prime}\left(N_{1}+N_{2}\right)\left(\int_{t-\rho_{2}}^{t} \dot{x}(s) d s\right)+2 \bar{\xi}^{\prime} N_{1}\left(\int_{t-\rho_{1}}^{t-\rho_{2}} \dot{x}(s) d s\right) \\
& \leq \rho_{2} \bar{\xi}^{\prime}\left(N_{1}+N_{2}\right)\left(R_{1}+R_{2}\right)^{-1}\left(N_{1}+N_{2}\right)^{\prime} \bar{\xi} \\
& +\int_{t-\rho_{2}}^{t} \dot{x}^{\prime}(s)\left(R_{1}+R_{2}\right) \dot{x}(s) d s \\
& +\left(\rho_{1}-\rho_{2}\right) \bar{\xi}^{\prime} N_{1} R_{1}^{-1} N_{1}^{\prime} \bar{\xi}+\int_{t-\rho_{1}}^{t-\rho_{2}} \dot{x}^{\prime}(s) R_{1} \dot{x}(s) d s, \\
& 2 \bar{\xi}^{\prime} N_{3}\left[\begin{array}{llll}
I & 0 & 0 & -I
\end{array}\right] \bar{\xi}=2 \bar{\xi}^{\prime} N_{3}\left(\int_{t-\tau_{\min }}^{t} \dot{x}(s) d s\right) \\
& \leq \tau_{\min } \bar{\xi}^{\prime} N_{3} R_{3}^{-1} N_{3}^{\prime} \bar{\xi}+\int_{t-\tau_{\min }}^{t} \dot{x}^{\prime}(s) R_{3} \dot{x}(s) d s \\
& 2 \bar{\xi}^{\prime} N_{4}\left[\begin{array}{llll}
0 & -I & 0 & I
\end{array}\right] \bar{\xi}=2 \bar{\xi}^{\prime} N_{4}\left(\int_{t-\rho_{1}}^{t-\tau_{\min }} \dot{x}(s) d s\right) \\
& \leq\left(\rho_{1}-\tau_{\min }\right) \bar{\xi}^{\prime} N_{4} R_{4}^{-1} N_{4}^{\prime} \bar{\xi}+\int_{t-\rho_{1}}^{t-\tau_{\min }} \dot{x}^{\prime}(s) R_{4} \dot{x}(s) d s,
\end{aligned}
$$

which relies on the fact that $x(t)-z_{1}(t)=x(t)-x\left(t-\rho_{1}\right)$ and $x(t)-z_{2}(t)=x(t)-x\left(t-\rho_{2}\right)$. The matrix variables $N_{1}, N_{2}, N_{3}, N_{4}$ represent degrees of freedom that can be exploited to minimize conservativeness and we call them slack matrices. Let us define $\beta:=\rho_{1}-\tau_{\min }$ and $\beta_{\max }:=$ $\rho_{1 \max }-\tau_{\min }$. Note that $\tau_{\min } \leq \rho_{1}-\rho_{2} \leq \tau_{\max }$ and

$$
\begin{aligned}
& \rho_{1 \max }=\sup _{k}\left(s_{k+1}+\tau_{k+1}-s_{k}+\tau_{k}-\tau_{k}\right) \leq \rho_{2 \max }+\tau_{\max }, \\
& \rho_{2 \max }+\tau_{\min }=\sup _{k}\left(s_{k+1}+\tau_{k+1}-s_{k}-\tau_{k}+\tau_{\min }\right) \leq \rho_{1 \max },
\end{aligned}
$$

so we conclude that $\tau_{\min } \leq \rho_{1 \max }-\rho_{2 \max } \leq \tau_{\max }, \rho_{2 \max } \leq$ $\beta_{\max }$, and $\rho_{2} \leq \beta$. After combining (21), (22),(23), and (24) and replacing $\rho_{2 \max }, \rho_{2}, \rho_{1}-\rho_{2}$ with $\beta_{\max }, \beta, \tau_{\max }$ we get

$$
\dot{V}(\bar{\xi}) \leq \bar{\xi}^{\prime}\left(\Psi+\beta_{\max }\left(M_{2}+M_{3}\right)+\beta\left(M_{4}-M_{3}\right)\right) \bar{\xi},
$$

where

$$
\begin{aligned}
\Psi & :=M_{1}+\tau_{\max } N_{1} R_{1}^{-1} N_{1}^{\prime}+\tau_{\min } N_{3} R_{3}^{-1} N_{3}^{\prime}, \\
M_{4} & :=\left(N_{1}+N_{2}\right)\left(R_{1}+R_{2}\right)^{-1}\left(N_{1}+N_{2}\right)^{\prime}+N_{4} R_{4}^{-1} N_{4}^{\prime},
\end{aligned}
$$

and $M_{1}, M_{2}, M_{3}$ are defined in (19). The necessary and sufficient condition to satisfy (25) is

$$
M_{1}+\tau_{M A T I}\left(M_{2}+M_{3}\right)<0, M_{1}+\tau_{M A T I}\left(M_{2}+M_{4}\right)<0
$$

(the proof is similar to the proof of Theorem 1 of [11]). By Schur complement, the matrix inequalities in (26), can be written as the LMIs in Theorem 2. It is easy to show that the Lyapunov functional satisfies the condition (3) with

$$
\psi_{1}(s):=d_{1} s^{2}, \quad \psi_{2}(\|\phi\|):=d_{2}|\phi(0)|^{2}+\bar{d}_{2} \int_{t-r}^{t}|\phi(s)|^{2} d s,
$$

for $d_{1}, d_{2}, \bar{d}_{2}>0$. If the LMIs in theorem 2 are feasible, then there exists a $d_{3}>0$ such that the condition (4) is satisfied with $\psi_{3}(s):=d_{3} s^{2}$ for $d_{3}>0$. The condition (5) is guaranteed by construction and consequently the system is GUES over $\mathscr{S}$.

\section{REFERENCES}

[1] R. Yuan, Z. Jing, and L. Chen;, "Uniform asymptotic stability of hybrid dynamical systems with delay," IEEE Trans. on Automat. Contr., vol. 48 , no. 2 , pp. 344 - 348, Feb 2003.

[2] X. Liu and J. Shen, "Stability theory of hybrid dynamical systems with time delay," IEEE Trans. on Automat. Contr., vol. 41, pp. 903-915, 2001.

[3] A. N. Michel, Y. Sun, and A. P. Molchanov, "Stability analysis of discontinuous dynamical systems determined by semigroups," IEEE Trans. on Automat. Contr., vol. 50, no. 9, pp. 1277-1290, Sep 2005.

[4] Y. Sun, A. N. Michel, and G. Zhai, "Stability of discontinuous retarted functional differential equations with applications," IEEE Trans. on Automat. Contr., no. 8, pp. 1090-1105, Aug 2005.

[5] J. P. Hespanha, P. Naghshtabrizi, and Y. Xu, "Networked control systems: analysis and design," Proc. of the IEEE, vol. 95, no. 1, pp. 138-162, Jan. 2007.

[6] G. C. Walsh, H. Ye, and L. Bushnell, "Stability analysis of networked control systems," IEEE Trans. on Contr. Syst. Tech., vol. 10, no. 3, pp. 438-446, May 2002.

[7] D. Nesic and A. R. Teel, "Input-output stability properties of networked control systems," IEEE Trans. on Automat. Contr., vol. 49, no. 10 , pp. 1650-1667, Oct. 2004.

[8] — , "Input-to-state stability of networked control systems," Automatica, vol. 40, no. 12, pp. 2121-2128, Dec 2004.

[9] E. Fridman, A. Seuret, and J. P. Richard, "Robust sample-data stabilization of linear systems: an input delay approach," Automatica, vol. 40, no. 8, pp. 1441-1446, Aug. 2004.

[10] D. Yue, Q.-L. Han, and C. Peng, "State feedback controller design for networked control systems," IEEE Trans. on Automat. Contr., vol. 51, no. 11, pp. 640-644, Nov. 2004.

[11] P. Naghshtabrizi, J. P. Hespanha, and A. R. Teel, "On the robust stability and stabilization of sampled-data systems: A hybrid system approach," in Proc. of the 45th Conf. on Decision and Contr., 2006, pp. 4873-4878.

[12] E. Fridman, "A new Lyapunov technique for robust control of systems with uncertain non-small delays," J. of Math. Contr. and Info., vol. 22, no. 3, Nov 2005.

[13] P. Naghshtabrizi and J. P. Hespanha, "Designing observer-based controller for network control system," in Proc. of the 44th Conf. on Decision and Contr., vol. 4, June 2005, pp. 2876-2880.

[14] D. Yue, Q. L. Han, and J. Lam, "Network-based robust $H_{\infty}$ control of systems with uncertainty," Automatica, vol. 41, no. 6, pp. 640-644, June 2005.

[15] W. Stallings, Data and Computer Communications, 6th ed. Prentice Hall, 2000.

[16] H. Khalil, Nonlinear systems, 2nd ed. Upper Saddle River, NJ: Prentice-Hall, 1996, page 204.

[17] W. Zhang, M. S. Branicky, and S. M. Phillips, "Stability of networked control systems," IEEE Contr. Syst. Mag., vol. 21, no. 1, pp. 84-99, Feb. 2001.

[18] M. Cloosterman, N. van de Wouw, W. Heemels, and H. Nijmeijer, "Robust stability of networked control systems with time-varying network-induced delays," in $c d c 06,2006$.

[19] M. Branicky, S. M. Philips, and W. Zhang, "Stability of networked control systems: explicit analysis of delay," in Proc. of the 2000 Amer. Contr. Conf., June 2000, pp. 2352-2357. 